\title{
Treatment of stroke patients in the context of the COVID-19 pandemic: Lessons learnt from a major stroke center in Vietnam
}

\section{Duy Ton Mai ${ }^{1 *}$, Hoang Phan ${ }^{1,2 *}$, Van Minh Hoang ${ }^{3}$, Tien Dung Nguyen ${ }^{1}$, Ha Quan Phan ${ }^{1}$, Xuan Trung Vuong ${ }^{1}$, Viet Phuong Dao ${ }^{1}$}

\author{
${ }^{1}$ Stroke Center, Bach Mai Hospital, Ha Noi, Vietnam \\ ${ }^{2}$ Menzies Institute for Medical Research, University of Tasmania, Tasmania, Australia \\ ${ }^{3}$ Hanoi University of Public Health, Hanoi, Vietnam \\ *Equal contribution
}

\begin{abstract}
T
\end{abstract} The coronavirus disease 2019 (COVID-19) pandemic is placing enormous strain on the global health care systems. The pandemic has led to a global decline in the number of stroke hospitalizations and interventions (eg, thrombolytic rate reduced by 13\%) [1]. The COVID-19 outbreak is continuing to spread around the world, with about 190 million confirmed cases and more than four million deaths across more than 200 countries [2]. For patients with acute stroke regardless of COVID-19 infection, evidence-based care is essential [3]. Country-specific strategies have been undertaken to respond to the pandemic to ensure access to treatment and care for stroke patients [4-6].

The COVID-19 pandemic has led to a global decline in the number of stroke hospitalizations and interventions.
The first COVID-19 infection was recorded in Vietnam on January 23, 2020. The country has entered the fourth wave of the COVID-19 pandemic from late-April 2021 with an exponential increase in the number of cases, mostly due to the rapid spread of new coronavirus variants. At the time of writing this paper (June 10, 2021), there had been 6595 cases confirmed in the last 45 days ( $n=4888$ in two provinces to the east of Hanoi: Bac Ninh and Bac Giang) out of a total of 8165 registered cases across the country since inception [7].

Bach Mai Hospital (BMH) is a major hospital in Hanoi, Vietnam. The stroke center at BMH has the largest catchment of patients across Northern Vietnam [8] with 800 stroke admissions per month pre-COVID. The center provides a wide range of evidence-based stroke care services, such as intravenous thrombolysis, mechanical thrombectomy, and coiling/clipping. During the fourth wave of the pandemic in Vietnam, the number of cases has been exponentially increasing, particular in Ha Noi and other surrounding provinces. To limit the spread of COVID-19, travel restrictions are in place across different cities and provinces in Vietnam. With the fast-changing situation, the risk of having F0 (the infected person), F1 (close contact with F0, or suspected of being infected), and F2 (close contact with F1) presented to our Stroke Center at BMH is inevitable. To avoid the risk of closing the Stroke Center, as was the situation in the $1^{\text {st }}$ wave of the pandemic [8], strategies have been implemented to maintain the best practice stroke care. 


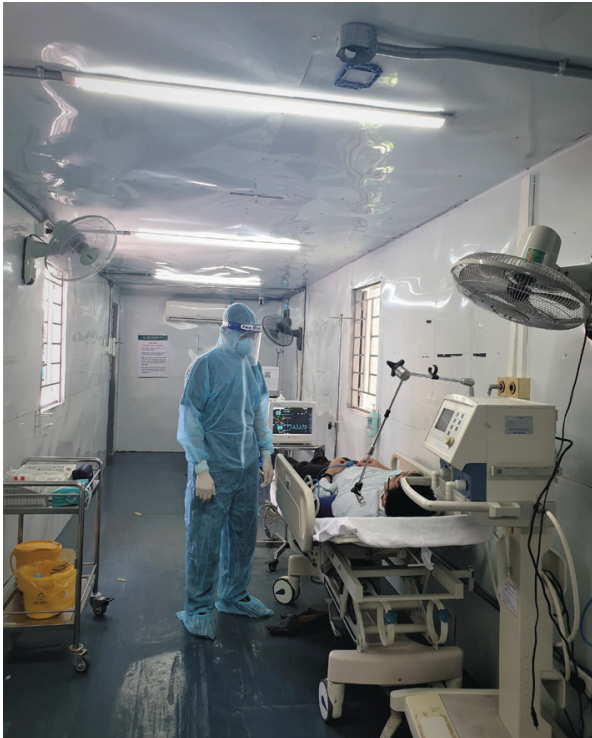

Photo: Treatment of stroke patients in the context of the COVID-19 pandemic at Bach Mai Hospital. Source: http:// bachmai.gov.vn/.
In this article, we share our experiences of the Stroke Center of BMH in the treatment of stroke patients in the context of the COVID-19 pandemic in Vietnam that could be useful for similar settings in other countries. Two main strategies have been employed to retain our stroke care services: 1) strengthening telemedicine to provide support for other hospitals that treat stroke; and 2) using a shipping-container Isolation Unit to maintain the treatment for stroke.

Our first approach is to strengthen telemedicine by using virtual consultations that connect $\sim 700$ stroke doctors from all hospitals across Northern Vietnam to pre-hospital triage patients with suspected stroke. During the $4^{\text {th }}$ wave, only those in need of urgent medical interventions should be admitted to the stroke center. This includes patients with 1) subarachnoid hemorrhages requiring coiling/clipping, 2) intracerebral or intraventricular hemorrhage for which an intervention is indicated. For ischemic stroke (IS), only those with large artery occlusion occurring within the 0-6 hours window and potentially eligible for mechanical thrombectomy are prioritized for admission to a stroke center. The patients must be tested for COVID-19 prior to transfer with results being reported to the telemedicine team during the transfer. Patients with an IS other than large artery occlusion are to be treated with intravenous thrombolysis, if eligible, under the guidance of virtual consultations with the telemedicine team at local hospitals.

We have developed a local protocol for triaging stroke patients during the pandemic (Figure 1). All the patients transferred from other hospitals or facilities should be presented to our Stroke Triage Unit, which is located outside the stroke center. Those who are F0, F1, or F2 are taken to the Stroke Isolation Unit (SIU) - a newly

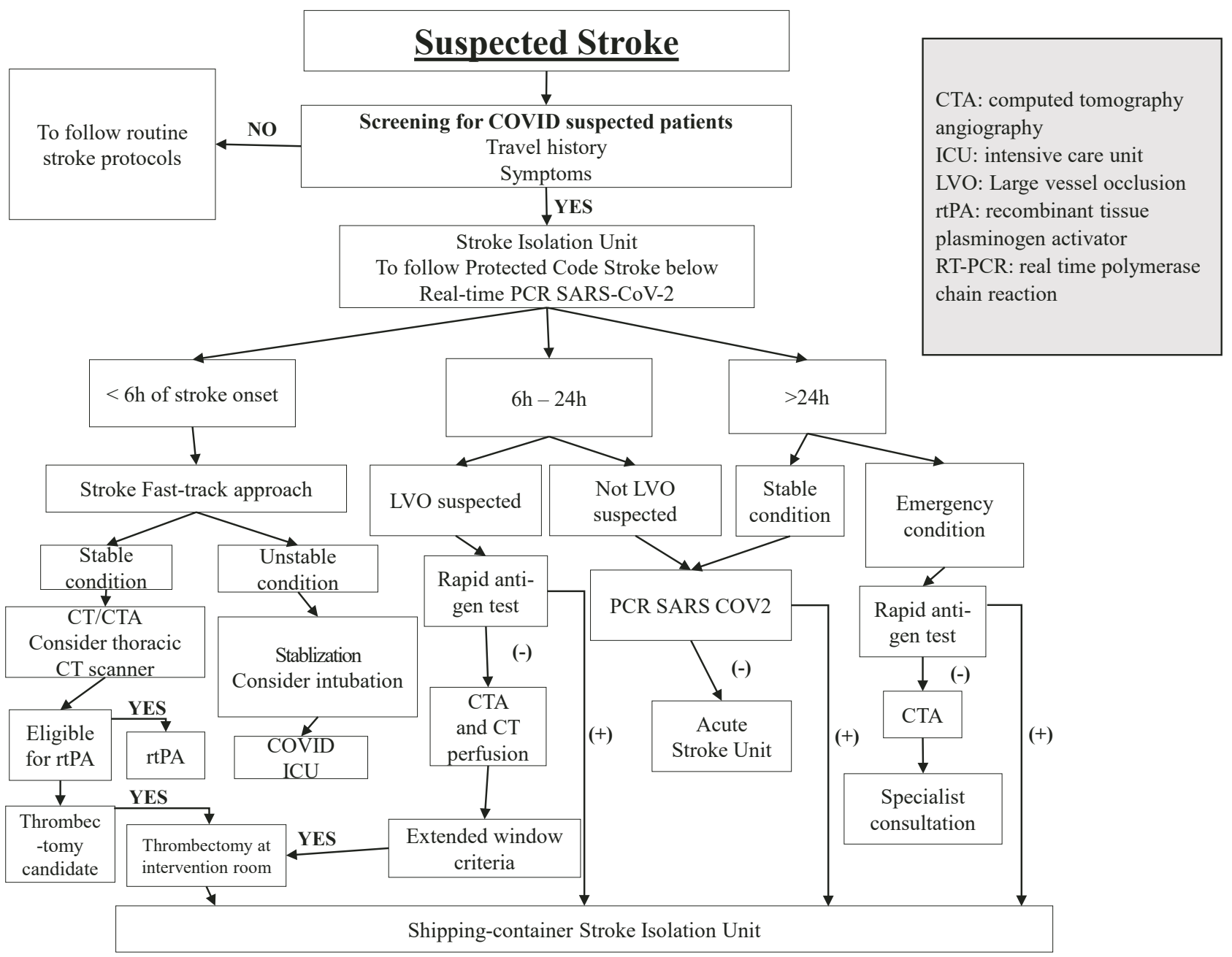

Figure 1. Flow-chart for triage stroke patients. 
We provide evidence that our strategies from a stroke center in Vietnam, including the use of telemedicine, triage protocols, and a shipping-container unit for isolation have brought benefits in maintaining best practice stroke care during the era of pandemic. established unit for further evaluation and treatment. The patients then have a brain imaging at the Department of Diagnostic Imaging and Intervention (DDII) at BMH using special arrangements for intra-hospital transfer (eg, via a special route of movement) and a designated intervention room (with restricted access) within the DDII for suspected COVID patients. If an intravenous thrombolysis is indicated, the patient is to be treated at the SIU. For those who are eligible and indicated, endovascular thrombectomy is performed within the DDII, with separate intervention rooms for COVID suspected patients and the remaining patients. All staff in the SIU are equipped with the highest level of transmission-based precautions, eg, personal protective equipment as well as walkie-talkie system for communications.

Our shipping-container SIU (Figure 2) was established on the April 28, 2021 to minimize the risk of a hospital cluster outbreak of COVID-19. The unit is equipped with air conditioning, oxygen, and compressed air systems for mechanical ventilation resuscitation. The cost for renting the shipping-container is about $175 \mathrm{USD} /$ month ( 4000000 Vietnam Dong). The mobile SIU was set up within 2 days in an isolated area within our stroke center to ensure cross-infection control of COVID-19 for frontline health care workers and patients.
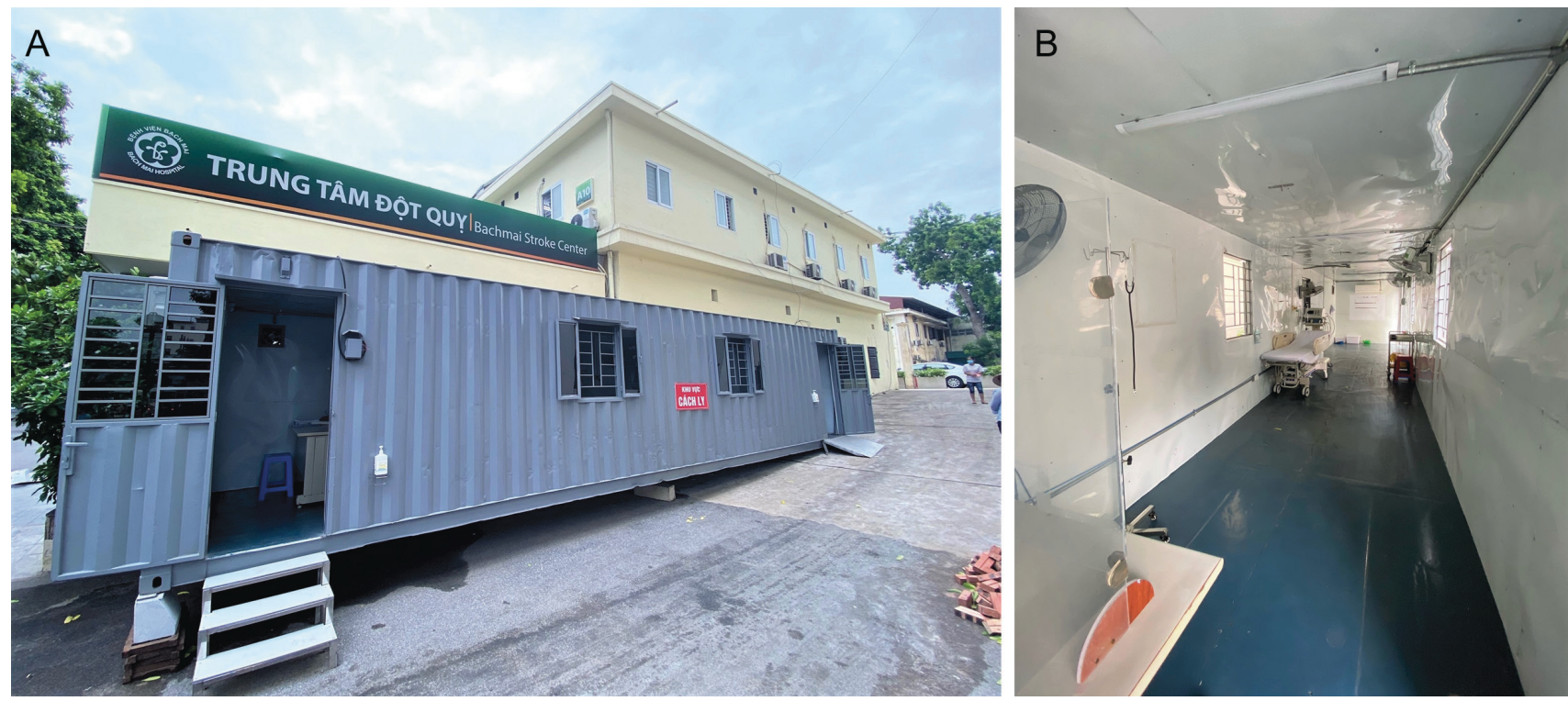

Figure 2. Shipping-container Stroke Isolation Unit at Bach Mai Hospital. Panel A. Front view. Panel B. Inside view.

Our local statistics show that the proportions of patients receiving reperfusion (IS) and clipping or coiling (hemorrhage stroke) were higher in May 2021 compared to the figures of earlier months (Table 1). This is likely due to the number of stroke admissions decreased significantly at 1 month following the $4^{\text {th }}$ wave occurrence. The potential reasons for the decline in stroke hospitalizations are a) patient fear of contracting COVID-19 [1], and b) physical distancing measures that may prevent people suffering stroke from timely seeking medical care [1], and c) only those in need of urgent medical interventions were hospitalized. The absolute number of interventions performed in May, although being smaller than that of the period preceding the pandemic wave, reflected our ability to maintain the best practice stroke care.

Table 1. Stroke admissions and intervention to the Stroke Center at Bach Mai Hospital in 2021

\begin{tabular}{|c|c|c|c|c|c|c|c|}
\hline & \multirow{2}{*}{$\begin{array}{c}\text { HOSPITAL } \\
\text { ADMISSIONS } \\
n\end{array}$} & \multicolumn{2}{|c|}{ STROKE CENTER ADMISSION } & \multicolumn{2}{|c|}{$\begin{array}{l}\text { REPERFUSION INTERVENTION PERFORMED } \\
\text { (THROMBOLYSIS/THROMBECTOMY) }\end{array}$} & \multicolumn{2}{|c|}{$\begin{array}{l}\text { NEUROSURGERY PERFORMED } \\
\text { (CLIPPING/COILING) }\end{array}$} \\
\hline & & $\mathrm{n}$ & $\%$ & $\mathrm{n}$ & $\%$ & $\mathrm{n}$ & $\%$ \\
\hline January & 13015 & 817 & 6.28 & 45 & 5.5 & 47 & 5.75 \\
\hline February & 5569 & 569 & 10.22 & 38 & 6.67 & 34 & 5.97 \\
\hline March & 12211 & 790 & 6.47 & 58 & 7.34 & 59 & 7.46 \\
\hline April & 12270 & 825 & 6.72 & 68 & 8.24 & 51 & 6.18 \\
\hline May & 5023 & 334 & 6.65 & 39 & 11.67 & 25 & 7.48 \\
\hline
\end{tabular}


Our shipping-container SIU at BMH is the first purpose-built unit for isolation of stroke patients with suspected COVID-19 to be used in a hospital setting in Vietnam. The findings provide evidence that our strategies, including the use of telemedicine, triage protocols, and the shipping-container SIU have brought benefits in maintain best practice care for patients with acute stroke at our center during the era of COVID pandemic. Our experiences could be useful for similar settings in other countries around the world.

Funding: Not declared.

Authorship contributions: All authors made substantial contributions to the study concept and design, acquisition of data, or data analysis, and interpretation of data. The authors also took part in drafting the opinion (DTM, HP and VPD), and/or and revising the manuscript critically for important intellectual content (the remaining authors); read and gave final approval of the version to be published; and agreed to be accountable for all aspects of the work (all authors).

Competing interests: The authors completed the ICMJE Disclosure of Interest Form (available upon request from the corresponding author), and declare no conflicts of interest.

1 Nogueira RG, Qureshi MM, Abdalkader M, Martins SO, Yamagami H, Qiu Z, et al. Global Impact of COVID-19 on Stroke Care and IV Thrombolysis. Neurology. 2021;96:e2824-38. Medline:33766997 doi:10.1212/WNL.0000000000011885

2 Johns Hopkins University COVID-19 Data Repository by the Center for Systems Science and Engineering (CSSE) at Johns Hopkins University. Available: https://arcg.is/OfHmTX. Accessed: 19 July 2021.

3 Smith MS, Bonomo J, Knight WA 4th, Prestigiacomo CJ, Richards CT, Ramser E, et al. Endovascular Therapy for Patients With Acute Ischemic Stroke During the COVID-19 Pandemic: A Proposed Algorithm. Stroke. 2020;51:00-00. Medline:32352910 doi:10.1161/STROKEAHA.120.029863

4 Venketasubramanian N, Anderson C, Ay H, Aybek S, Brinjikji W, de Freitas GR, et al. Stroke Care during the COVID-19 Pandemic: International Expert Panel Review. Cerebrovasc Dis. 2021;50:245-61. Medline:33756459 doi:10.1159/000514155

5 Markus HS, Martins S. COVID-19 and stroke-Understanding the relationship and adapting services. A global World Stroke Organisation Perspective. Int J Stroke. 2021;16:241-7. Medline:33709834 doi:10.1177/17474930211005373

6 Douiri A, Muruet W, Bhalla A, James M, Paley L, Stanley K, et al. Stroke Care in the United Kingdom During the COVID-19 Pandemic. Stroke. 2021;52:2125-33. Medline:33896223 doi:10.1161/STROKEAHA.120.032253

7 Vietnam Ministry of Health. Health Portal on Coronavirus Disease (COVID-19) of Vietnam. Available: https://ncov.moh.gov. vn/. Accessed: 10 June 2021.

8 Nguyen QT, Hoang TA, Nguyen HYT, Xuan CD, Thanh Do V, Dong VT, et al. Prevent and control cross-transmission of COVID-19 in hospital settings: Lessons learned from a national hospital in Hanoi, Vietnam. J Glob Health. 2021;11:03079. Medline:34131485 doi:10.7189/jogh.11.03079

\author{
Correspondence to: \\ Hoang T Phan \\ Stroke Center \\ Bach Mai Hospital \\ 78 Giai Phong \\ Dong Da District \\ Ha Noi \\ Vietnam \\ thi.phan@utas.edu.au
}

